\title{
SIMULTANEOUS MOMENTUM, HEAT AND MASS TRANSFER WITH CHEMICAL REACTION IN A DISORDERED POROUS MEDIUM: APPLICATION TO BINDER REMOVAL FROM A CERAMIC GREEN BODY
}

\author{
GREGORY C. STANGLE and ILHAN A. AKSAY \\ Department of Materials Science and Engineering, and Advanced Materials Technology Center, \\ Washington Technology Centers, University of Washington, Seattle, WA 98195, U.S.A.
}

(First received 27 December 1988; accepted in revised form 4 December 1989)

\begin{abstract}
A theoretical model has been developed to describe simultaneous momentum, heat, and mass transfer phenomena in disordered porous materials. The model can be applied to a wide variety of engineering-related fields, e.g., the drying and/or burnout of processing aids in the colloidal processing of advanced ceramic materials. Simulations based on the model predict the local temperature and mass distribution of the porous body as a function of time and position. This information can then be coupled with known mechanical properties of the body to predict internal stresses generated during removal of liquid from the body. The theoretical model has potential application to many engineering problems, e.g., the optimization of processing conditions in the design of an improved binder removal process. The model is evaluated using experimental data on binder removal from a ceramic green compact consisting of submicron $\alpha-\mathrm{Al}_{2} \mathrm{O}_{3}$ powder dispersed in a paraffin wax; the agreement between the simulated and experimental results is good.
\end{abstract}

\section{INTRODUCTION}

Simultaneous momentum, heat, and mass transfer in a porous medium is an important subject in a large number of engineering fields. The situation often arises in which it is necessary to remove liquid from a porous solid by evaporation and/or chemical reaction. Numerous interesting and significant examples exist in the literature, including the drying of granular materials (including ceramics), wood products, food products, and soil. Often the saturated material is placed in an external drying medium (usually a flowing gas stream) and the liquid undergoes phase change by either (i) evaporation or (ii) chemical reaction followed by desorption. The gaseous species are then carried away by the external medium. If this liquid removal process is conducted too quickly, however, damage to the porous object can occur. The warping, cracking and blistering of objects, for example, during the drying of dimensional lumber (Plumb et al., 1985) and the removal of binder components from ceramic green compacts illustrate the significance of such a complication. The permanent deformation of materials during liquid removal is due to internal stresses generated during removal of the liquid. A slow rate of removal, on the other hand, requires the input of significant amounts of time and energy that may cause the process to be no longer cost-effective.

Modeling of the process can provide a sound basis for process design considerations, thereby helping to quantify many of the factors involved in carrying out the process successfully. The process to be modeled involves a three-phase system: an inert porous solid phase, a liquid phase, and a multicomponent gas phase. The model should predict the relative fraction and relative movement of each phase within the sample as a function of position and time. Temperature profiles must be taken into account in nonisothermal processes. The internal stress profiles (as will be shown in later sections) depend upon the microstructure (i.e., the spatial arrangement of solids and pores) of the sample as well as the liquid saturation profile (defined as the volume fraction of the pore space filled with liquid) within the sample. These interrclationships require the simultaneous incorporation of transport processes and mechanical properties into a single model describing the process.

Existing models of heat and mass transfer in porous media are generally concerned with the evaporation of water from rigid porous materials (e.g., sand or sandstone). These models usually take into account some subset of the following transport phenomena: capillary flow of liquid, convective flow and diffusion of gas, and heat transfer by conduction and convection within the body. Most published theoretical investigations have focused primarily on drying phenomena and can be categorized (Berger and Pei, 1973) into four groups: diffusion theory (Sherwood, 1931), capillary flow theory (Ceaglske and Hougen, 1937), evaporation-condensation theory (Gurr et al., 1952), and the application of irreversible thermodynamics (Luikov, 1966). These models tend either to be incomplete in their treatment of all transport processes that occur during at least one portion of the drying process or include expressions (e.g., Luikov, 1966) that are difficult to obtain or interpret experimentally. As a result, these approaches usually fall short of describing the dynamic phenomena taking place in porous materials during the removal of the liquid phase. A return to a more fundamental description of simultaneously occurring transport phenomena (Bird et al., 1960 ) is more appropriate and has been considered by 
more recent work (Whitaker, 1977; see also Whitaker and Chou, 1983; Plumb et al., 1985).

The theoretical treatment of liquid removal from a porous solid must also include a consideration of the mechanical properties of the material, especially when it is known that the material is not so rigid that permanent deformation can occur under certain circumstances. Porous granular materials (soils, ceramics) can be particularly prone to damage during the liquid removal process. In general, this aspect requires full consideration of the viscoelastic properties of the material over a relatively wide range of strains and strain rates to which the material is to be subjected. If the deformations are small and the rate of deformation is low, elasticity theory (Timoshenko and Goodier, 1970) might be applied as a first approximation to the description of the mechanical behavior of porous granular material during the process of liquid removal.

The model described here considers simultaneous momentum, heat and mass transfer with chemical reaction and evaporation in a disordered porous medium. The model takes into account liquid transport via capillary action, gas transport by convection and diffusion, and phase change by chemical reaction and evaporation. Effective transport coefficients are estimated based on percolation concepts as applied to disordered media. External heat and mass transfer coefficients are variable in order to account for changing conditions external to the porous body. Internal stress distributions are determined through the use of an approach to obtain local stresses in partially saturated porous media, combined with elasticity theory. Profiles of temperature, gas- and liquidphase hold-up, gas-phase composition, and stress are predicted. Model predictions are compared with experimentally obtained results.

\section{THEORETICAL MODEL}

The theoretical model developed in this section is based upon the following physical observations: (a) the solid phase consists of small, discrete grains whose size is much smaller than the size of the body; (b) the liquid phase, which consists of a single component, flows within the sample due to a gradient in liquidphase pressure, which in turn is due to a gradient in liquid saturation; (c) phase change of liquid to gas occurs either by evaporation or by liquid-phase chemical reaction followed by desorption; (d) gas transport can occur either by diffusion under a concentration or partial pressure gradient, or by convection under a total gas-phase pressure gradient; (e) for evaporation only, the partial pressure of the evaporating liquidphase species in the presence of condensed liquid is considered to be at equilibrium saturation partial pressure at all times; (f) heat transfer within the body takes place by conduction and by convection of bulk liquid and gas phases; (g) local thermal equilibrium is assumed to be established in length scales much smaller than the size of the body but much larger than the size of an individual solid grain (a continuum approximation); (h) effective transport coefficientsliquid and gas permeabilities, thermal conductivity, gas-phase diffusivity - are estimated by applying percolation concepts to knowledge of the body's microstructure and transport properties of the individual phases; (i) heat and mass transfer at the surface of the body occur because of differences of temperature and partial pressure, respectively, between the surface and the external medium; (j) the gas phase is considered to be a mixture of ideal gases; (k) viscous dissipation, compressional work, and body forces other than gravity are negligible; (1) pressure, forced and thermal diffusion (Bird et al., 1960) are much smaller than concentration diffusion and are thus neglected; and (m) the deformation of the granular porous matrix arises from internal stresses generated during liquid removal, due primarily to saturation-weighted phase pressures (McTigue et al., 1983) of which gas-phase and capillary pressures are most significant.

The model, based in part upon basic concepts of transport phenomena, involves the simultaneous solution of the appropriate momentum, energy, and material continuity equations. Boundary conditions make use of the usual symmetry and continuity of flux conditions. The particular form (see, e.g., Whitaker and Chou, 1983) of the boundary conditions for the liquid phase depends upon the value of the liquidphase permeability coefficient (which is zero at low liquid saturation values), while those for the gas phase also depend upon liquid saturation, since partial pressure of the evaporating species is no longer the equilibrium partial pressure at zero liquid saturation. External heat and mass transfer coefficients are determined from standard methods (Bird et al., 1960).

\section{A. Transport equations}

The appropriate transport equations and initial and boundary conditions are given here in a spherical coordinate system in order to facilitate comparison with experimental data obtained for a spherical object (Rhee et al., 1990). Details of the comparison are given in a following section. The energy continuity equation is given by

$$
\begin{array}{r}
\rho C_{\mathrm{p}} \frac{\partial T}{\partial t}+\left[\rho_{\mathrm{L}} C_{\mathrm{pL}} v_{\mathrm{L}}+\rho_{\mathrm{G}} C_{\mathrm{pG}} v_{\mathrm{G}}\right] \frac{\partial T}{\partial r}+\Delta h_{\mathrm{v}} \dot{m}_{\mathrm{e}} \\
=\frac{1}{r^{2}} \frac{\partial}{\partial r}\left[r^{2} K_{\mathrm{T}}^{\mathrm{E}} \frac{\partial T}{\partial r}\right]+\sum_{\kappa}\left(-\Delta H_{\kappa}\right) r_{\kappa}
\end{array}
$$

where $T$ is temperature, $r$ is radial position, $t$ is time, $\rho$ is density, $C_{p}$ is heat capacity, $v_{x}$ is radial velocity of phase " $x$," $K_{\mathrm{T}}^{\mathrm{E}}$ is effective thermal conductivity, $\dot{m}_{\mathrm{c}}$ is evaporation rate (with enthalpy of phase change $\Delta h_{v}$ ), and $r_{\kappa}$ is the liquid decomposition (to gas) reaction rate (with enthalpy of reaction, $\Delta H_{\kappa}$ ). The liquid is considered to be single-component, but decomposes to form a number of gaseous species. Subscripts "L" and "G" indicate liquid and gas phases, respectively. 
The momentum balance for the liquid phase is

$$
v_{\mathrm{L}}=-\frac{\boldsymbol{K}_{\mathrm{L}}^{\mathrm{E}}}{\mu_{\mathrm{L}}}\left[\frac{\partial \boldsymbol{P}_{\mathrm{L}}}{\partial r}-\rho_{\mathrm{L}} g\right]
$$

where $K_{\mathrm{L}}^{\mathrm{E}}$ is effective liquid phase permeability, $\mu$ is viscosity, and $P$ is pressure, while the momentum balance for the gas phase is

$$
v_{\mathrm{G}}=-\frac{K_{\mathrm{G}}^{\mathrm{E}}}{\mu_{\mathrm{G}}}\left[\frac{\partial P_{\mathrm{G}}}{\partial r}-\rho_{\mathrm{G}} g\right]
$$

where $K_{G}^{\mathrm{E}}$ is the effective gas phase permeability. Further, the continuity equation for the liquid phase is

$$
\frac{\partial}{\partial t}\left(\rho_{\mathrm{L}} \varphi_{\mathrm{L}}\right)+\frac{1 \partial}{r^{2} \partial r}\left(\rho_{\mathrm{L}} r^{2} v_{\mathrm{L}}\right)+\dot{m}_{\mathrm{e}}+\sum_{\kappa} r_{\kappa}=0
$$

where $\varphi_{x}$ is volume fraction of phase " $x$," and the continuity equation for the gas phase is

$$
\frac{\partial}{\partial t}\left(\varphi_{G} \rho_{G}\right)+\frac{1 \partial}{r^{2} \partial r}\left(\rho_{G} r^{2} v_{G}\right)-\dot{m}_{e}-\sum_{\kappa} r_{\kappa}=0 .
$$

Finally, the diffusion equations for the gas-phase components are given by

$$
\begin{aligned}
& \frac{\partial}{\partial t}\left(\varphi_{G} \rho_{G i}\right)+\frac{1 \partial}{r^{2} \partial r}\left(\rho_{G i} r^{2} v_{G}\right)-\dot{m}_{i}-r_{i} \\
& \quad=\frac{1 \partial \partial}{r^{2} \partial r}\left[r^{2} \rho_{G} D^{E} \frac{\partial}{\partial r}\left(\frac{\rho_{G i}}{\rho_{G}}\right)\right] \quad 1 \leqslant i \leqslant(N-1)
\end{aligned}
$$

where $D^{\mathrm{E}}$ is the effective diffusivity and subscript " $i$ " indicates component " $i$ ".

These equations are subject to the following initial and boundary conditions for a spherically symmetric sample. Initially,

$$
\begin{gathered}
t=0, \quad 0 \leqslant r \leqslant r_{\mathrm{p}}, \quad T=T_{0}, \\
\varphi_{\mathrm{L}}=\varphi_{\mathrm{LO}}, \rho_{\mathrm{G} t}=\rho_{\mathrm{G} i \mathrm{O}} .
\end{gathered}
$$

The symmetry condition applies at the center of the sample:

$$
\begin{gathered}
t>0, \quad r=0, \quad \frac{\partial T}{\partial r}=0 \\
\frac{\partial \varphi_{\mathrm{L}}}{\partial r}=0, \quad \frac{\partial \rho_{\mathrm{Gi}}}{\partial r}=0
\end{gathered}
$$

while the surface $\left(r=r_{p}\right)$ boundary conditions assume the following form (for $t>0$ ):

$$
\begin{gathered}
K \mathrm{E} \frac{\partial T}{\partial r}+\Delta h_{\mathrm{v}} \dot{M}_{\mathrm{S}}+h_{\mathrm{e}}\left(T-T_{\infty}\right)=0 \\
\rho_{\mathrm{L}} V_{\mathrm{L}}+\rho_{\mathrm{G} i} V_{\mathrm{G}}+\rho_{\mathrm{G}} D^{\mathrm{E}} \frac{\partial}{\partial r}\left(\frac{\rho_{\mathrm{G} i}}{\rho_{\mathrm{G}}}\right) \\
-h_{m}\left(X_{\mathrm{Gi}}-X_{\mathrm{Gi} i}\right)=0 \\
P_{\mathrm{G}}=P_{\mathrm{m} t \mathrm{~m}}
\end{gathered}
$$

where $\dot{M}_{\mathrm{S}}$ is the total evaporation rate at the surface of the sample, $h_{\mathrm{c}}$ and $h_{\mathrm{m}}$ are external heat and mass transfer coefficients, respectively, and $X_{G i}$ is the mass function of component " $i$ " in the gas.

\section{B. Effective transport coefficients}

The effective transport coefficients $\left(K\right.$ T, $K_{\mathrm{L}}^{\mathrm{E}}, K_{\mathrm{G}}^{\mathrm{E}}$ and $\left.D^{E}\right)$ in eqs (1)-(3) and (6), respectively, must incorporate microstructural features in order to be employed in a quantitative description of the process. For example, when the thermal conductivity of the solid phase is much higher than those of the interstitial fluids in the porous body, the solid matrix (which may simply be a collective of a large number of discrete solid particles in contact with neighboring particles) can provide a sort of "preferred pathway" for heat conduction. Similarly, gas-phase transport by convection or diffusion occurs only through a connected network of pores devoid of liquid. Further, liquid transport by convection in a porous body is possible when the liquid phase is hydraulically connected - that is, through a connected network of liquid-filled pores. A wide variety of pore space and "nonpore" space models have been used in this regard and are summarized in the review by Van Brakel (1975). Many represent an undesirable oversimplification of the actual physical situation and often require "fudge factors" (Scheidegger, 1954) to provide reasonably accurate values for the transport coefficients.

Among the available theories that incorporate a physically realistic description of the pore network geometry and topology and provide useful numerical values of effective transport coefficients, percolation concepts as applied to disordered porous media have exhibited great promise (Mohanty et al., 1982; Reyes and Jensen, 1985). The concept of connectedness inherent in these approaches represents a marked improvement over the simpler representations of "straight-through" pores described by Van Brakel (1975). In addition, percolation theories offer to modeling efforts the advantage of computational savings with respect to that which would be required for a complete characterization of the pore network.

In the present work, the Bethe lattice (or Cayley tree) is used to approximate the pore space in the porous granular body. Stinchcombe (1974) laid the groundwork for this approach, in which he allowed for a continuous distribution of local coefficients for sites and bonds in the network (i.e., pore bodies and pore necks, respectively, in the case of a pore network). Reyes and Jensen (1985) used Stinchcombe's results directly in the determination of effective diffusion coefficients within a porous solid, using this approach later in the modeling of gas-solid reactions (Reyes and Jensen, 1986a, b). Stinchcombe's (1974) useful analytical form of equations for estimating effective transport coefficients will be employed in this work as well, resulting in an estimation of liquid and gas permeabilities as well as gas-phase diffusivities. A brief synopsis of the pertinent results is given below. 
According to percolation theory for a Bethe lattice, the effective transport coefficient, $K^{\mathrm{E}}$, is given by:

$$
K^{\mathrm{E}}=\varepsilon \bar{k}_{i}
$$

where

$$
\varepsilon=-\left(\frac{z-1}{z-2}\right) \frac{C^{\prime}(0)}{\bar{k}_{i}}
$$

and $\bar{k}_{i}$ is some (unspecified) reference value of $k_{i}$. [Note that combination of eqs (14) and (15) gives $K^{\mathrm{E}}$ in terms of $z$ and $C^{\prime}(0)$, thus eliminating the need to know $\bar{k}_{i}$.] Now, for $\left(\varphi_{\mathrm{L}}-\varphi_{\mathrm{LC}}\right)<1 / z$,

$$
\varepsilon=1.522 \frac{(z-1)}{(z-2)^{2}} \frac{\left\langle k_{i}(r)\right\rangle}{k_{i}}\left(\frac{\varphi_{L}-\varphi_{L C}}{\varphi_{L C}}\right)^{2}
$$

with

$$
\left\langle k_{i}(r)\right\rangle=\left\{\int_{0}^{\infty} \frac{F\left(k_{i}(r)\right)}{k_{i}(r)} \mathrm{d} k_{i}(r)\right\}^{-1}
$$

and

$$
\boldsymbol{F}\left(k_{i}(r)\right)=\frac{f(r) \mathrm{d} r}{\mathrm{~d} k_{i}}(\boldsymbol{r})
$$

where $f(r)$ is the pore size distribution, given here by the following relationship (see also Dullien, 1979):

$$
f(r)=c\left[\frac{r-r_{\min }}{r_{\max }-r_{\min }}\right]^{a}\left[1-\frac{r-r_{\min }}{r_{\max }-r_{\min }}\right]^{b}
$$

where $c$ is subject to the normalization condition $\int_{0}^{\infty} f(r) \mathrm{d} r=1$.

$$
\begin{aligned}
& \text { For }\left(\varphi_{\mathrm{L}}-\varphi_{\mathrm{LC}}\right)>1 / z, \text { define } \\
& \qquad G[k(r)] \equiv\left(1-\varphi_{\mathrm{L}}\right) \delta[k(r)]+\varphi_{\mathrm{L}} F[k(r)]
\end{aligned}
$$

and

$$
H[k(r)] \equiv \frac{k(r)(z-1) C^{\prime}(0)}{k(r)-(z-1) C^{\prime}(0)} .
$$

$C^{\prime}(0)$ is obtained by iteration for eq. (15) from the following implicit expression:

$$
\begin{aligned}
& 0=\int_{0}^{\infty} G[k(r)]\left\{H[k(r)]-C^{\prime}(0)\right\} \mathrm{d} k(r) \\
& +(z-1) I_{210} \alpha_{2}^{0} \\
& \times\left[1+(z-1) I_{220}\right]+(z-1) I_{310} \alpha_{3}^{0} \\
& +I_{410}\left[(z-1)\left(\alpha_{2}^{0}\right)^{2}+(z-1) \alpha_{4}^{0}\right] \\
& +\left(\begin{array}{c}
z-1 \\
2
\end{array}\right) I_{510} \alpha_{2}^{0} \alpha_{3}^{0}+\left(\begin{array}{c}
z-1 \\
3
\end{array}\right) I_{610}\left(\alpha_{2}^{0}\right)^{3}
\end{aligned}
$$

in which

$$
\begin{aligned}
\alpha_{n}^{0}= & \int_{0}^{\infty} \frac{G[k(r)]\left\{H[k(r)]-C^{\prime}(0)\right\}^{n} \mathrm{~d} k(r)}{n !} \\
I_{m n o}= & \frac{m !}{(n-1) !} \int_{0}^{\infty} \\
& \times \frac{G[k(r)][k(r)]^{2}\left\{H[k(r)]-C^{\prime}(0)\right\}^{n-1} \mathrm{~d} k(r)}{\left[k(r)-(z-1) C^{\prime}(0)^{m+1}\right]}
\end{aligned}
$$

and

$$
\left(\begin{array}{l}
n \\
k
\end{array}\right)={ }_{n} C_{k}=\frac{n !}{(n-k) ! k !}
$$

The quantities required for carrying out the calculations include the pore-level value of transport coefficients and the pore size distribution information. The local transport coefficients are based upon the assumption of locally straight cylinders, giving, for the liquid permeability, $k_{i}=r_{s i}^{2} / 8$, for the gas permeability, $k_{i}=r_{b i}^{2} / 8$, and for the gas diffusivity:

$$
\frac{1}{k_{i}}=\frac{1}{D_{e, j}}=\frac{1}{D_{\mathrm{K}_{j}}}+\frac{1}{D_{j, \mathrm{~m}}}
$$

where

$$
\begin{gathered}
D_{\mathrm{K} j}=\frac{4}{3} r_{b}\left(\frac{2}{\pi} \cdot \frac{R T}{M_{j}}\right)^{1 / 2} \\
\frac{1}{D_{j, \mathrm{~m}}}=\frac{\sum_{k=1}^{N} \frac{1}{D_{j k}}\left(y_{k}-y_{j} \cdot \frac{N_{k}}{N_{j}}\right)}{1-y_{j} \sum_{k=1}^{N} N_{k} / N_{j}}
\end{gathered}
$$

where eqs (27) and (28) are the Knudsen and concentration diffusion coefficients, respectively. Note that $N_{j}$ and $N_{k}$ are scalar quantities for this one-dimensional problem.

Note that the site (pore body) distribution was used for $K_{\mathrm{L}}^{\mathrm{E}}$, while the bond (pore neck) distribution was used for $K_{\mathrm{G}}^{\mathrm{E}}$ and $D^{\mathrm{E}}$. The rationale for this is discussed in more detail by Ramakrishnan and Wasan (1986). The values of $a$ and $b$ are estimated from the results of Chatzis and Dullien (1985) for a porous solid made up of monosized spheres (1/4 and 1 , respectively).

The effective thermal conductivity coefficient, $K_{T}^{\mathrm{E}}$, can be obtained quite simply from the work of Batchelor and O'Brien (1977) for conduction through a random assemblage of monosized spheres:

$$
K_{\mathrm{T}}^{\mathrm{E}}=\frac{1}{2} k_{\mathrm{F}} \varphi_{\mathrm{S}} \bar{Z} \log \left(\alpha^{2}\right)
$$

such that $\alpha=k_{\mathrm{s}} / k_{\mathrm{F}}$ and the average number of contact points, $\bar{Z}$, by (Ridgeway and Tarbuk, 1967):

$$
\Phi=1.072-0.1153 \bar{Z}+0.00431 \bar{Z}^{2} .
$$

It should be noted that this expression for $K_{\mathrm{T}}^{\mathrm{E}}$ is appropriate for cases such that $\alpha \gg 1$ and $\varphi_{\mathrm{S}} \geqslant 0.60$. Other expressions are available for lower volume fractions $\left(\varphi_{\mathrm{s}}=0.3-0.5\right)$, that is for two- or three-phase systems in which the relatively high conductivity phase is discrete and in which relatively few interparticle contacts are made (Nozad et al., 1985a, b). For ceramic green bodies considered here, typical ranges of $\varphi_{\mathrm{S}}$ and $\alpha$ are $0.58-0.64$ and $30-100$, respectively.

\section{Capillary pressure and stress distribution}

The capillary pressure in a partially saturated porous medium, defined as $P_{\mathrm{C}}=P_{\mathrm{G}}-P_{\mathrm{L}}$, is known to be a function of liquid content, $\varphi_{\mathrm{L}}$ (Scheidegger, 1974; DeWiest, 1969; and others). Since capillary pressure, 
as defined by the Laplace equation,

$$
P_{\mathrm{C}}=\frac{2 \sigma \cos \theta}{R}
$$

depends upon the radius of liquid-filled pores within the porous medium, which in turn is a function of $\varphi_{L}$, the capillary pressure can be estimated to a first approximation in the following way. Assuming (Scheidegger, 1974; DeWiest, 1969) that all pores of radius less than $\bar{R}$ are filled with liquid, then $\bar{R}$ is related to the saturation by:

$$
\varphi_{\mathrm{L}}=\frac{\int_{0}^{\bar{R}} V(r) f(r) \mathrm{d} r}{\int_{0}^{\infty} V(r) f(r) \mathrm{d} r} .
$$

The value of $\bar{R}$ acquired in eq. (32) is then employed in eq. (31) to obtain the local value of $\boldsymbol{P}_{C}$ for the given local value of $\varphi_{\mathbf{L}}$.

Finally, the stress distribution within the porous body that is generated during liquid removal can be obtained through application of elasticity theory (Timoshenko and Goodier, 1970). This analysis, of course, is subject to the limitation of a small degree of deformation and a low rate of deformation-a situation that arises, for example, during binder removal from ccramic compacts with high particlc packing densities. Now, for a partially saturated granular medium, McTigue et al. (1983) have shown that the effective local stress is given by

$$
\sigma_{\mathrm{E}}=\sigma_{\mathrm{S}}+P_{\mathrm{G}^{\prime}}-\varphi_{\mathrm{L}} P_{\mathrm{C}^{\prime}}
$$

where $\sigma$ and $\imath$ are second-order stress and unit tensors, respectively. Timoshenko and Goodier (1970) show that in an elastic solid the local strain must be corrected to account for the presence of surrounding material, relative to that in the absence of surrounding material. Equation (33), therefore, accounts only for free strain (actually the strain corresponding to the local stress, which for an elastic solid body are linearly related) and must be corrected to give the net local stresses as

$$
\boldsymbol{\sigma}=\sigma_{\mathrm{E}}-\overline{\boldsymbol{\sigma}}
$$

where

$$
\bar{\sigma}=\frac{1}{V_{\mathrm{p}}} \int_{0}^{V_{\mathrm{p}}} \sigma_{\mathrm{E}} \mathrm{d} V .
$$

Thus the net local stresses arise from differences between the local value of unrestrained ("free") stress and the average value throughout the body in the case for which material properties (i.e., Young's modulus, $E$, and Poisson's ratio, $v$, of the bone-dry porous body) are constant, thus preserving the linear relationship between stress and strain.

Since the solutions to the material and energy balance equations described above are solved in their spherical coordinate form (as discussed in the following section), it is convenient to resolve the total stress, $\sigma=\sigma(r)$, into its radial and tangential components.
Following Timoshenko and Goodier (1970):

$$
\begin{aligned}
& \sigma_{r}(r)=\frac{2 E}{1-v}\left[\frac{1}{r_{\mathrm{p}}^{3}} \int_{0}^{r_{\mathrm{p}}} \sigma(r) r^{2} \mathrm{~d} r-\frac{1}{r^{3}} \int_{0}^{r} \sigma(r) r^{2} \mathrm{~d} r\right] \\
& \sigma_{\theta}(r)=\frac{E}{1-v}\left[\frac{2}{r_{\mathrm{p}}^{3}} \int_{0}^{r_{\mathrm{p}}} \sigma(r) r^{2} \mathrm{~d} r+\frac{1}{r^{3}} \int_{0}^{r} \sigma(r) r^{2} \mathrm{~d} r-\sigma(r)\right] .
\end{aligned}
$$

Thus the resolved components of stress in the body during liquid removal depend upon the instantaneous local values of gas pressure, capillary pressure, and liquid saturation-values obtainable through solution of the transport equations.

\section{NUMERICAL SOLUTION}

To make and verify theoretical predictions, a large number of physical, kinetic, and thermodynamic properties must be known or estimated in order to obtain the solution to the simultaneous equations (1)-(6). Further, verification of the theoretical predictions with experimental results is possible only when actual physical properties are available and included in the calculations. As an example, the removal of nonceramic materials from a ceramic green compact consisting of submicron $\alpha-\mathrm{Al}_{2} \mathrm{O}_{3}$ powder in paraffin wax will be considered. The green body is a sort of composite of ceramic powder (discrete phase) and wax (continuous phase). The physical properties for this system are given in Table 1 . The shape of the green body considered here is spherical (for comparison with Rhee et al., 1990): it is therefore a sphere composed of many small spheres, bound together by paraffin wax. For binder removal, the green compact is placed in a flowing gas stream of predetermined composition in a temperature-controlled oven. The ratio of oven diameter to green body diameter (R hee et al., 1990 ) is roughly 35 , indicating that the green body is positioned in a large medium. The temperature history of the oven is also specified. The heat and mass transfer coefficients used in eqs (11) and (12) depend on conditions in the oven and are given (Bird et al., 1960) for a sphcre (grcen body) in a flowing gas stream.

$$
\begin{aligned}
\frac{h_{e} d_{\mathrm{p}}}{k_{\mathrm{G}}} & =2.0+0.60\left(\frac{d_{\mathrm{p}} V_{\mathrm{o}} \rho_{\mathrm{G}}}{\mu_{\mathrm{G}}}\right)_{\mathrm{f}}^{1 / 2}\left(\frac{C_{\mathrm{pG}} \mu_{\mathrm{G}}}{k_{\mathrm{G}}}\right)_{\mathrm{r}}^{1 / 3} \\
\frac{h_{\mathrm{m}} d_{\mathrm{p}}}{c_{\mathrm{F}} D_{\mathrm{ABf}}} & =2.0+0.60\left(\frac{d_{\mathrm{p}} V_{\mathrm{o}} \rho_{\mathrm{G}}}{\mu_{\mathrm{G}}}\right)_{\mathrm{f}}^{1 / 2}\left(\frac{\mu_{\mathrm{G}}}{\rho_{\mathrm{G}} D_{\mathrm{AB}}}\right)_{\mathrm{f}}^{1 / 3} .
\end{aligned}
$$

To facilitate the numerical solution of the nonlinear eqs (1)-(6), the following variables are defined:

$$
\begin{aligned}
\Theta & =\frac{T-T_{0}}{T_{F}-T_{0}} \\
U & =\frac{\varphi_{\mathrm{L}}}{\varphi_{\mathrm{LO}}} \\
W & =\frac{\rho_{\mathrm{GN}}}{\rho_{\mathrm{GNO}}}
\end{aligned}
$$


Table 1. Physical properties required for simulations

Solid $\left(\mathrm{Al}_{2} \mathrm{O}_{3}\right)$

$$
\begin{aligned}
\rho_{\mathrm{s}} & =3980 \\
k_{\mathrm{s}} & =4.18 \\
C_{\mathrm{ps}} & =270,605+2.758 \times 10^{-3} T-8.482 \times 10^{5} / T^{2} \\
\bar{Z} & =0.1193-(\sqrt{0.0172} \Phi-0.0043) / 0.0086 \\
\varphi_{\mathrm{s}} & =0.63
\end{aligned}
$$

Liquid (pentacosane, $\mathrm{C}_{25} \mathrm{H}_{52}$ )

$$
\begin{aligned}
\rho_{\mathrm{L}} & =780\left[\left(T_{\mathrm{C}}-T\right) /\left(T_{\mathrm{C}}-T_{\mathrm{r}}\right)\right]^{0.29} \\
T_{\mathrm{C}} & =826 \\
k_{\mathrm{L}} & =0.02806 C_{\mathrm{pL}} \rho_{\mathrm{L}}^{1.33} / T \\
C_{\mathrm{PL}} & =2317.8 \\
\mu_{\mathrm{L}} & =\left(\rho_{\mathrm{L}}^{1 / 2} \cdot 10^{8}\right) / 8569.8+1.398\left(T_{\mathrm{C}}-T\right) / T
\end{aligned}
$$

Interface

$$
\begin{aligned}
\sigma & =\sigma_{1}\left[\left(1-T_{\mathrm{r}}\right) /\left(1-T_{\mathrm{r} 1}\right)\right]^{1.2}, \quad T_{\mathrm{r}}=T / T_{\mathrm{C}} \\
\sigma_{1} & =0.0308 \\
\theta & \rightarrow 0
\end{aligned}
$$

Gas-pentacosane

$$
\begin{aligned}
P_{\mathrm{G} 1}= & 10^{\beta, \beta}=-4548.3 / T+9.734 \\
\rho_{\mathrm{G} 1}= & P_{\mathrm{Gl}} M_{1} / R T \\
\mu_{1}= & 352 \\
C_{\mathrm{pG} 1}= & 10.291+5.342 \times 10^{-1} T-2.924 \times 10^{-4} T^{2} \\
& +6.2 \times 10^{-4} T^{3} \\
\mu_{\mathrm{G} 1}= & 0.000327\left(T / T_{C}\right)^{0.94} \\
k_{\mathrm{G} 1}= & 2.133 \times 10^{-1} C_{\mathrm{pG}}(0.0176 T-0.3324)^{0.667} \\
\mathrm{Gas}-\mathrm{N}_{2} & \\
\rho_{\mathrm{GN}}= & P_{\mathrm{GN}} M_{\mathrm{N}} / R T \\
C_{\mathrm{pGN}}= & 6.5+0.001 T \\
\mu_{\mathrm{GN}}= & 4 \times 10^{-5}(T-323)+0.019 \\
k_{\mathrm{GN}}= & 0.0065(T-300)+2.59
\end{aligned}
$$

$$
\begin{aligned}
\tau & =\frac{\alpha_{\mathrm{s}} t}{r_{\mathrm{p}}^{2}} \\
R & =\frac{r}{r_{\mathrm{p}}} \\
\alpha_{\mathrm{s}} & =\frac{k_{\mathrm{s}}}{\rho_{\mathrm{s}} C_{\mathrm{ps}}} .
\end{aligned}
$$

Now, for a spherically symmetric sample, the dimensionless form of eqs (1) (6), after substantial rearrangement, become in a spherical coordinate system:

$$
\begin{gathered}
\frac{\partial \Theta}{\partial \tau}+A_{1} \frac{\partial \Theta}{\partial R}+A_{2} \frac{\partial^{2} \Theta}{\partial R^{2}}+A_{3}=0 \\
\frac{\partial\left(U \rho_{\mathrm{L}}\right)}{\partial \tau}+A_{4} \frac{\partial U}{\partial R}+A_{5} \frac{\partial^{2} U}{\partial R^{2}}+A_{6}=0 \\
\frac{\partial\left(\varphi_{\mathrm{G}} W\right)}{\partial \tau}+A_{7} W+A_{\mathrm{g}} \frac{\partial W}{\partial R}+A_{9} \frac{\partial^{2} W}{\partial R^{2}}=0 \\
\dot{m}_{\mathrm{e}}=A_{10}
\end{gathered}
$$

where:

$$
\begin{aligned}
A_{1}= & \frac{1}{\rho C_{\mathrm{p}} \alpha_{\mathrm{s}}} \times\left[\frac{\rho_{\mathrm{L}} C_{\mathrm{pL}} K_{\mathrm{L}}^{\mathrm{E}}}{\mu_{\mathrm{G}}} \frac{\partial P_{\mathrm{c}}}{\partial R}-\frac{\rho_{\mathrm{L}} C_{\mathrm{pL}} K_{\mathrm{L}}^{\mathrm{E}}}{\mu_{\mathrm{L}} R} \frac{\partial P_{\mathrm{G}}}{\partial R}\right. \\
& \left.-\frac{\rho_{\mathrm{G}} C_{\mathrm{pG}} K_{\mathrm{G}}^{\mathrm{E}}}{\mu_{\mathrm{G}}}-\frac{\partial P_{\mathrm{G}}}{\partial R} \frac{\partial K_{\mathrm{T}}^{\mathrm{E}}}{\partial R}-\frac{2 K_{\mathrm{T}}^{\mathrm{E}}}{R}\right]
\end{aligned}
$$

$$
\begin{aligned}
& \boldsymbol{A}_{2}=-\frac{K_{\mathrm{T}}^{\mathrm{E}}}{\rho C_{\mathrm{p}} \alpha_{\mathrm{S}}} \\
& A_{3}=\frac{r_{\mathrm{p}}^{2} \Delta h_{\mathrm{v}}}{\left(T_{\mathrm{F}}-T_{\mathrm{o}}\right) \rho C_{\mathrm{p}} \alpha_{\mathrm{S}}} \dot{m}_{\mathrm{c}}-\frac{r_{\mathrm{p}}^{2}}{\left(T_{\mathrm{F}}-T_{\mathrm{o}}\right) \rho C_{\mathrm{p}} \alpha_{\mathrm{S}}} \sum_{\mathrm{k}} r_{\kappa} \\
& A_{4}=\frac{\rho_{\mathrm{L}}}{\varphi_{\mathrm{L}} \alpha_{\mathrm{S}}}\left[\frac{2 K_{\mathrm{L}}^{\mathrm{E}} k_{\varphi}}{\mu_{\mathrm{L}} R}+\frac{\partial}{\partial R}\left(\frac{K_{\mathrm{L}}^{\mathrm{E}} k_{\varphi}}{\mu_{\mathrm{L}}}\right)\right] \\
& A_{s}=\frac{K_{\mathrm{L}}^{\mathrm{E}} k_{\varphi} \rho_{\mathrm{L}}}{\alpha_{\mathrm{s}} \mu_{\mathrm{L}} \varphi_{\mathrm{L}}} \\
& A_{6}=\frac{\rho_{\mathrm{L}}}{\varphi_{\mathrm{Lo}} \alpha_{\mathrm{S}}}\left[-\frac{\partial}{\partial R}\left(\frac{K_{\mathrm{L}}^{\mathrm{E}}}{\mu_{\mathrm{L}}}\right) \frac{\partial P_{\mathrm{G}}}{\partial R}-\frac{2 K_{\mathrm{L}}^{\mathrm{E}}}{\mu_{\mathrm{L}}} \frac{1}{R} \frac{\partial P_{\mathrm{G}}}{\partial R}\right. \\
& -\frac{K_{\mathrm{L}}^{\mathrm{E}}}{\mu_{\mathrm{L}}} \frac{\partial^{2} \boldsymbol{P}_{\mathrm{G}}}{\partial R^{2}}+\frac{\partial}{\partial R}\left(\frac{\boldsymbol{K}_{\mathrm{L}}^{\mathrm{E}} k_{\mathrm{T}}}{\mu_{\mathrm{L}}}\right) \frac{\partial \Theta}{\partial R}+\frac{K_{\mathrm{L}}^{\mathrm{E}} k_{\mathrm{T}}}{\mu_{\mathrm{L}}} \frac{1}{R} \frac{\partial \Theta}{\partial R} \\
& \left.+\frac{K_{\mathrm{L}}^{\mathrm{E}} k_{\mathrm{T}}}{\mu_{\mathrm{L}}} \frac{\partial^{2} \Theta}{\partial R^{2}}+\frac{r_{\mathrm{p}}^{2}}{\rho_{\mathrm{L}}} \dot{m}_{\mathrm{e}}\right] \\
& A_{7}=\frac{1}{\alpha_{\mathrm{S}}}\left[\frac{-1}{\mu_{\mathrm{G}}} \frac{\partial K_{\mathrm{G}}^{\mathrm{E}}}{\partial R} \frac{\partial P_{\mathrm{G}}}{\partial R}-\frac{2 K_{\mathrm{G}}^{\mathrm{E}}}{\mu_{\mathrm{G}}} \frac{1}{R} \frac{\partial P_{\mathrm{G}}}{\partial R}-\frac{K_{\mathrm{G}}^{\mathrm{E}}}{\mu_{\mathrm{G}}} \frac{\partial^{2} P_{\mathrm{G}}}{\partial R^{2}}\right. \\
& \left.+\frac{1}{\rho_{\mathrm{G}}} \frac{\partial D^{\mathrm{E}}}{\partial R} \frac{\partial \rho_{\mathrm{G}}}{\partial R}+\frac{2 D^{\mathrm{E}}}{\rho_{\mathrm{G}}} \frac{1}{R} \frac{\partial \rho_{\mathrm{G}}}{\partial R}+\frac{D^{\mathrm{E}}}{\rho_{\mathrm{G}}^{2}} \frac{\partial^{2} \rho_{\mathrm{G}}}{\partial R^{2}}\right] \\
& A_{\mathrm{B}}=\frac{1}{\alpha_{\mathrm{S}}}\left[\frac{-K_{\mathrm{E}}^{\mathrm{G}}}{\mu_{\mathrm{G}}} \frac{\partial P_{\mathrm{G}}}{\partial R}-\frac{\partial D^{\mathrm{E}}}{\partial R}-\frac{2 D^{\mathrm{E}}}{R}+\frac{D^{\mathrm{E}}}{\rho_{\mathrm{G}}} \frac{\partial \rho_{\mathrm{G}}}{\partial R}\right] \\
& A_{9}=\frac{-D^{\mathrm{E}}}{\alpha_{\mathrm{s}}} \\
& A_{10}=\frac{\alpha_{\mathrm{s}}}{r_{\mathrm{p}}^{2}} \frac{\partial\left(\varphi_{\mathrm{G}} \rho_{\mathrm{G}}\right)}{\partial \tau}+\frac{1}{r_{\mathrm{p}}^{2}}\left[\frac{-\partial P_{\mathrm{G}}}{\partial R} \frac{K_{\mathrm{G}}^{\mathrm{E}}}{\mu_{\mathrm{G}}} \frac{\partial \rho_{\mathrm{G}}}{\partial R}\right. \\
& -\frac{\rho_{\mathrm{G}}}{\mu_{\mathrm{G}}} \frac{\partial K_{\mathrm{G}}^{\mathrm{E}}}{\partial R} \frac{\partial P_{\mathrm{G}}}{\partial R}-\frac{2}{R} \frac{\rho_{\mathrm{G}} K_{\mathrm{G}}^{\mathrm{E}}}{\mu_{\mathrm{G}}} \frac{\partial P_{\mathrm{G}}}{\partial R} \\
& \left.-\frac{\rho_{G} K_{G}^{E}}{\mu_{G}} \frac{\partial^{2} P_{G}}{\partial R^{2}}\right] \text {. }
\end{aligned}
$$

After eqs (1)-(6) were nondimensionalized, they were then discretized in order to employ a fully implicit finite difference solution technique (Carnahan et al., 1969). This set of equations with appropriately discretized initial and boundary conditions forms a tridiagonal set of simultaneous equations that are simply solved by the Thomas method for tridiagonal matrix inversion (Carnahan et al., 1969). The equations for $\Theta, U, W$, and $\dot{m}_{e}$ [eqs (46)-(49)] are solved simultaneously at each time step based on values at the previous time step; the coefficients $A_{1}-A_{10}$ are calculated for values at the previous step and are thus considered constant at the current time step. The instantaneous local values of radial and tangential stresses are normalized with respect to the local stress calculated at an arbitrarily chosen value of liquid saturation, i.e., when local liquid saturation reaches the percolation threshold. For the Bethe lattice, this is $\varphi_{\mathrm{L}}=1 /(z-1)$. For the case when $\left|P_{\mathrm{G}}\right| \ll\left|\varphi_{\mathrm{L}} P_{\mathrm{C}}\right|$ and $\left|\sigma_{\mathrm{s}}\right| \ll\left|\varphi_{\mathrm{L}} P_{\mathrm{C}}\right|$, this normalization factor remains constant for a given microstructural configuration of pores and solids. Precise knowledge of $E$ and $v$ are thus not required. 


\section{RESULTS AND DISCUSSION}

The theoretical model eqs (46)-(49), along with coefficients $A_{1}-A_{10}$ given by eqs (50)-(59), were solved subject to the initial and boundary conditions given by eqs (7)-(13). The calculations gave temperature, liquid-gas, and gas composition profiles in the porous body as a function of position and time. This information was then used to calculate stress distribution profiles, also as a function of position and time. The results were compared with experimental results reported in a companion paper (Rhee et al., 1990) for the removal of molten binder from an injection molding mixture. Based upon successful validation of the model by comparison with experimental data, numerical calculations were then conducted to simulate additional liquid removal situations. These calculations can provide a sound basis for the design of additional experimental work and can be used to provide an understanding of various binder removal schemes.

Figure 1 represents a comparison between the predictions based upon the model presented here and the experimental results of Rhee et al. (1990). The sample from which the liquid was removed consisted of submicron $\alpha-\mathrm{Al}_{2} \mathrm{O}_{3}$ particles and paraffin wax. The samples $\left(r_{\mathrm{p}}=0.002 \mathrm{~m}\right)$ were placed in the sample pan of a thermogravimetric analysis (TGA) apparatus and subjected to the following temperature regimen: the samples were heated at a specified linear rate of temperature increase $\left(10^{\circ} \mathrm{C} / \mathrm{min}\right)$ to a final temperature, $T_{\mathrm{f}}$, which was held constant for the duration of the experiment. Sample weight was monitored at frequent time intervals. The computer simulations were carried out to reflect experimental conditions and those results are represented by the solid lines in Fig. 1. Gas to liquid phase change was considered to be evaporation only, so that $r_{k}=0$ for all $\kappa$. Initial reaction kinetic studies were reported by Stangle et al. (1989), thus substantiating that evaporation predominates over chemical reaction. Very good agreement between experimental and predicted results is seen, except for the case of $T_{\mathrm{f}}=195^{\circ} \mathrm{C}$ after $120 \mathrm{~min}$. This is probably due to the choice of the value of the coordination number for the

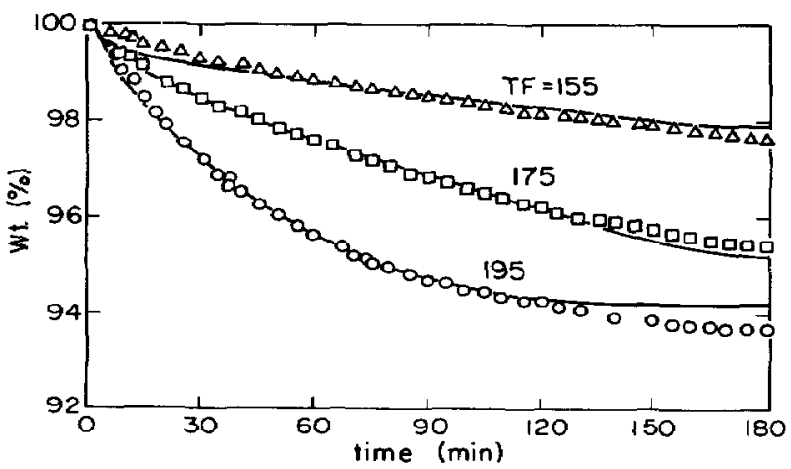

Fig. 1. Comparison of theoretical predictions with experimental results of $\mathrm{Rhee}$ et al. (1989).
Bethe lattice representation of the pore network ( $z$ =7), which determines the percolation threshold for liquid permeability. (At earlier times, however, the predicted weight loss was shown in a sensitivity study to be unaffected by the choice of $z$ for $z \geqslant 5.0$.) As will be apparent in the following paragraphs, this alters the predominant mechanism by which material transport occurs within the porous body: that is, capillary liquid flow at early times is replaced by diffusion and convection of gaseous components at later stages. Since the former mechanism is responsible for a greater rate of weight loss than is the latter, a value of the coordination number that incorrectly represents the percolation threshold will lead to less accurate predictions at low values of liquid saturation, $U$. Parameter sensitivity studies were also conducted for the following quantities in eq. (19), the expression for the pore size distribution, $f(r): a, b, r_{\min }$ and $r_{\text {max }}$. This is perhaps the most significant point at which to perform such a sensitivity analysis, since the transport coefficients $\left(K_{\mathrm{L}}^{\mathrm{E}}, K_{\mathrm{G}}^{\mathrm{E}}\right.$, and $\left.D^{\mathrm{E}}\right)$ and capillary pressure $\left(P_{\mathrm{C}}\right)$ depend directly upon $f(r)$. In all instances, any variation in $a, b, r_{\min }$ or $r_{\max }$ that resulted in a shift of the mean pore size yielded a sizeable difference in weight loss rate, internal stress levels, and material flux values. It should be noted, however, that even small shifts in the mean pore size are difficult to achieve experimentally (Rhee et al., 1990) for samples at this solids-volume fraction $(>60 \mathrm{v} / \mathrm{o})$ and particle size $(0.8 \mu \mathrm{m})$, so that deviations of parameter values from values appropriate to this system must be considered to be less representative of the system studied experimentally. In general, however, the model developed here may be considered to be successful in its prediction of sample weight loss in the alumina-paraffin system.

The model was employed to predict temperature, mass, and stress profiles in samples from which liquid is removed under conditions similar to those described in the preceding paragraph, and subsequent to verification. Thus, simulations specified a period of linear temperature increase, followed by a period of constant temperature ("isothermal"). Sample weight (expressed as a percentage of original weight) versus dimensionless time, $\tau$, was predicted. The results for four values of $T_{\mathrm{f}}$ are shown in Fig. 2. The differences in the curves for different values of $T_{\mathrm{f}}$ are due to a large extent to $P_{1}^{0}$ (and to a lesser extent upon $h_{\mathrm{m}}$ and $h_{\mathrm{e}}$ ), which depends upon $T_{\mathrm{f}}$ after the initial heating up (or "induction") period has been completed and $T_{\mathrm{f}}$ has been reached. The curves for $T_{f}=155^{\circ} \mathrm{C}$ and $230^{\circ} \mathrm{C}$ represent extreme cases since liquid removal (for a ceramic/binder system, for example) would probably be considered too slow to be economically important in the former case and would be undesirable because of cracking in the latter. Thus, they serve as good standards to which other binder removal schemes might be compared.

Figures 3 and 4 illustrate an important difference in liquid saturation profiles between these two temperature histories. For $T_{\mathrm{f}}=155^{\circ} \mathrm{C}$ (Fig. 3), the liquid satu- 


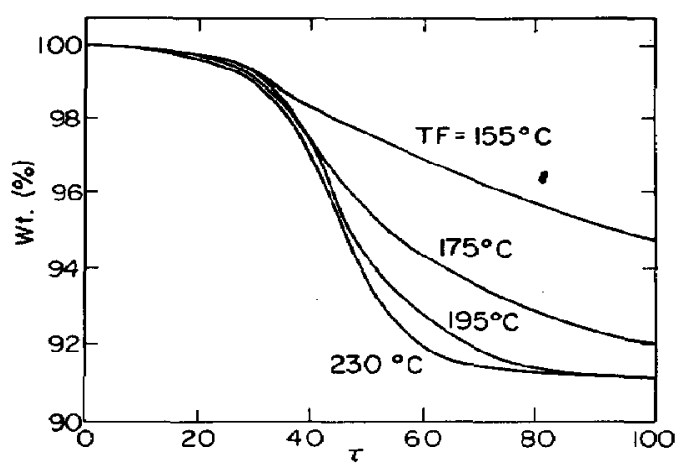

Fig. 2. Simulated sample weight loss vs. time for four "isothermal" heating conditions.

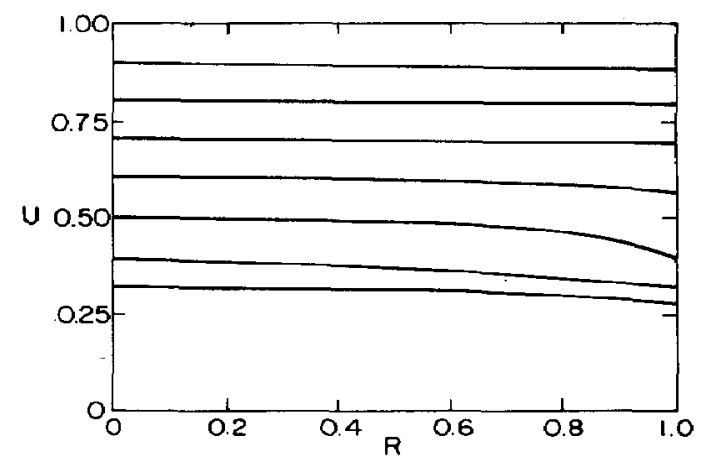

Fig. 3. Liquid saturation profiles for isothermal heating conditions: $T_{\mathrm{f}}=155^{\circ} \mathrm{C}$.

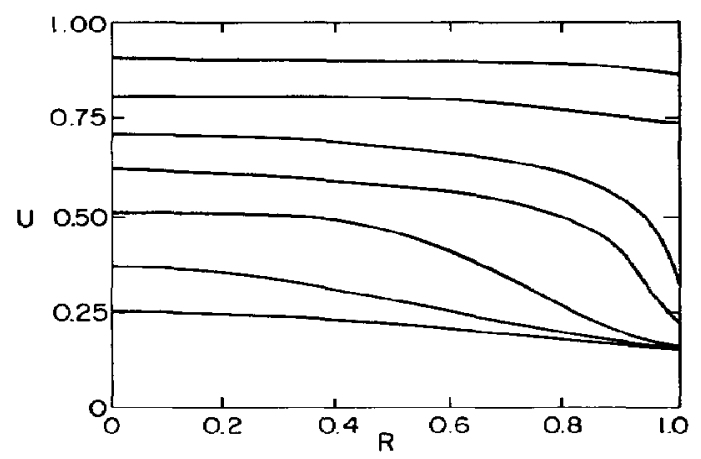

Fig. 4. Liquid saturation profiles for isothermal heating conditions: $T_{\mathrm{f}}=230^{\circ} \mathrm{C}$.

ration decreases steadily as a function of time and evenly as a function of position. For $T_{\mathrm{f}}=230^{\circ} \mathrm{C}$, on the other hand, Fig. 4 shows predictions of nonuniform saturation and thus relatively steep liquid gradients, particularly between $U(0) \cong 0.70$ and $U(0) \cong$ 0.40 , where $U(0)$ is the value of $U$ at the center of the sample. The difference between the two liquid saturation profiles may be explained in the following manner. Liquid within the solid must be gasified in order to be removed from the body. This may take place at the sample surface, where the mass transfer rate from the surface is given by the last term in eq. (12), $h_{\mathrm{m}}\left(X_{\mathrm{G} i}-X_{\mathrm{Gi} \infty}\right)$, and where $X_{\mathrm{G} i}$ increases significantly with temperature. Alternatively, liquid-to-gas phase change may take place within the solid, after which it is transported through empty pores to the external gas medium by diffusive and convective mechanisms. Order of magnitude comparisons of calculated values of these various contributions to material transport show that surface evaporation is the only dominant mechanism for approximately the first $70 \%$ of the liquid removal (for $\bar{U}<0.3$, i.e., during the final stages of liquid removal, internal evaporation becomes increasingly important). Thus, liquid is removed more quickly from the surface of the sample at $T_{\mathrm{f}}=230^{\circ} \mathrm{C}$ than at $155^{\circ} \mathrm{C}$, due primarily to the higher vapor pressure. The liquid saturation gradients steepen in the $230^{\circ} \mathrm{C}$ case, however, since calculations show that liquid permeability decreases sharply as liquid saturation decreases, thereby reducing the liquid phase velocity, $v_{\mathrm{L}}$, as given by eq. (2). This decrease in $K_{\mathrm{L}}^{\mathrm{E}}$ through the range of $0.4 \leqslant \bar{U} \leqslant 1$ is greater than the increase in $P_{\mathrm{C}}$ through the same range. The net result is that the region near the sample's surface for the case of $T_{\mathrm{f}}=230^{\circ} \mathrm{C}$ loses liquid by surface evaporation more quickly than it can be replenished from the interior of the sample. At $T_{\mathrm{f}}=155^{\circ} \mathrm{C}$, on the other hand, liquid transport from the sample interior nearly matches the rate at which surface evaporation takes place.

Predictions concerning gas- and liquid-phase pressure distributions within the porous body were then employed to determine stress distribution generated during the process. Equations (33)-(37) were employed to calculate the radial and tangential stress profiles that correspond to the calculated distribution of individual phases within the sample. Figure 5 shows the predicted profiles of the normalized stress components, $\sigma_{r}^{*}$ and $\sigma_{\theta}^{*}$, for an "isothermal" temperature history with $T_{\mathrm{f}}=230^{\circ} \mathrm{C}$. Curves (a) and (b) correspond to a relatively early time in the process $[U(0)=0.9]$, while curves (c) and (d) are those for the

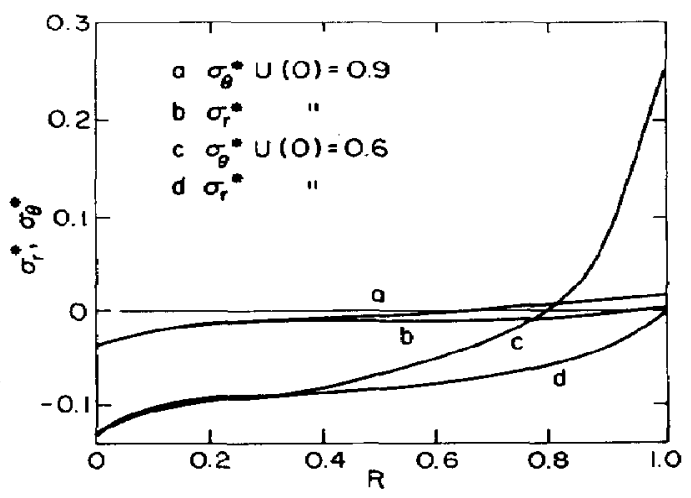

Fig. 5. Radial and tangential stress profiles during binder removal for isothermal heating conditions: $T_{\mathrm{f}}=230^{\circ} \mathrm{C} . U(0)$ designates the value of liquid saturation of the center of the sample. 
middle portion of the process $[U(0)=0.6]$. The convention used here is that, for example, a positive value of $\sigma^{*}(R)$ indicates that the portion of the body at $R$ is locally under tension, while $\sigma^{*}(R)<0$ indicates compression at $R$. It should be noted that radial stresses are always in compression, as opposed to the tangential stresses that are in tension near the surface of the body $(R>0.7)$ and in compression elsewhere. This is significant since it is known (Sherrington and Oliver, 1981) that partially saturated granular materials are much weaker in tension than in compression and that failure (that is, cracking and other permanent microstructural deformations) can occur if any component of stress locally exceeds the tensile strength of the material. Figure 6(a) and (b) shows in more detail the evolution of the tangential component of stress at various values of liquid saturation. The maximum tensile stress occurs at the surface of the body $(R=1)$ and is seen to grow from small values during the early portion of the liquid removal process $[U(0)=0.9]$ to a maximum for the middle portion $[U(0) \cong 0.6]$ and thereafter to decay to smaller values. This maximum in $\sigma_{\theta}^{*}$ at $R=1$ corresponds to the sharp liquid saturation gradients in Fig. 4, indicating the importance of maintaining "flat" profiles in $U$ in order to minimize internal stresses that may damage

(a)

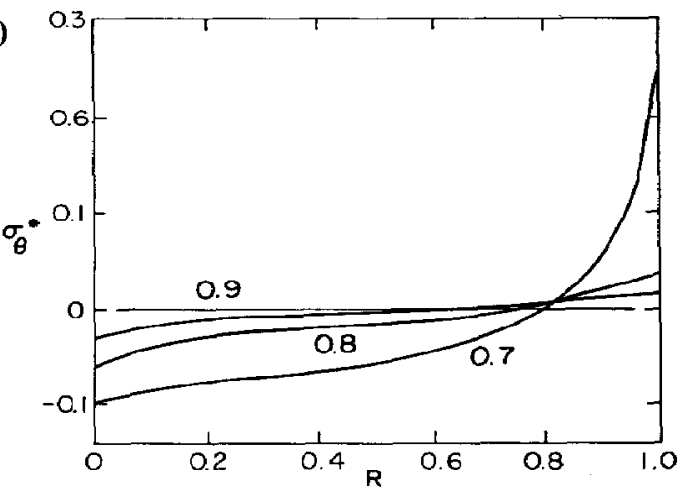

(b)

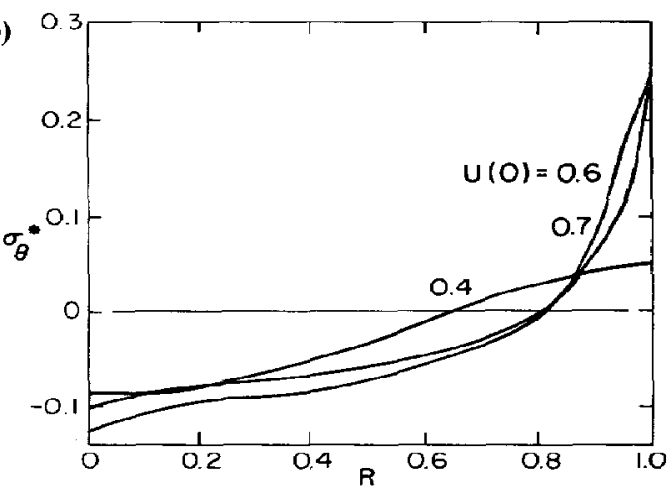

Fig. 6. Tangential stress profiles at various times [given here for successively smaller values of $U(0)]$. Isothermal heating conditions $\left(T_{\mathrm{f}}=230^{\circ} \mathrm{C}\right)$ were specified. (a) Profiles for a nearly saturated sample in the range of $1.0 \geqslant U(0) \geqslant 0.7$. (b)

Profiles for the less saturated range $0.7 \geqslant U(0) \geqslant 0.4$.

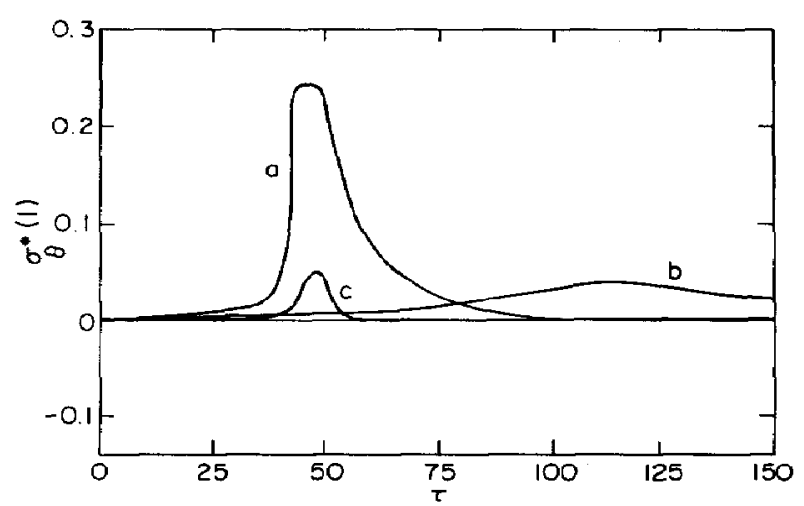

Fig. 7. Surface tangential stress history of sample for various heating conditions.

the sample. Figure 7 represents this relationship in another form, displaying graphically the relative magnitude of this surface tangential stress component and the time at which the large stresses arise during the liquid removal processes. Curves (a) and (b) illustrate the significant differences in tensile stress build-up and decay for $T_{\mathrm{f}}=230^{\circ} \mathrm{C}$ and $155^{\circ} \mathrm{C}$, respectively.

This analysis of stress distribution profiles can be extended to include evaluation of binder removal from ceramic compacts as practiced industrially. The first such process involves a process by which the ceramic compact is caused to lose its liquid at a linear weight loss rate, while the second involves the reduction of liquid-phase viscosity in order to minimize the magnitude of internal stress within the body. Johnsson et al. (1984) described a binder removal process, termed "rate-controlled extraction," by which the temperature in the furnace is adjusted to ensure a constant weight loss rate throughout most of the binder removal process. This is accomplished with a controller that monitors sample weight and adjusts the oven temperature accordingly. Results of the calculation are shown in Fig. 8, for a representative value of specified weight loss rate. The maximum value of $\sigma_{\theta}^{*}(1)$ is approximately 0.23 (which differs little from the value for the "isothermal" heating case of $T_{\mathrm{f}}=230^{\circ} \mathrm{C}$ ) and occurs at approximately the dimensionless time $\tau=55$. Further, oven temperatures as high as $340^{\circ} \mathrm{C}$ are required in the attempt to maintain the linear weight loss rate. Thus, little reduction in maximum stress was predicted and much higher temperatures, relatively, were required by this method. The stress distribution in Fig. 8 is rather unexpected in that two peaks are predicted. It should be remembered, however, that the control scheme is to adjust the ambient temperature, $T_{\infty}$, so that a linearity in the weight-loss rate is maintained (until $\tau \cong 70$ when the principal mass transport mechanism shifts to gasphase diffusion). The two peaks in $\sigma_{\theta}^{*}(1)$ represent an obviously undesirable overshooting of an uncontrolled quantity. Clearly, the wrong variable is being controlled: a more clever approach would be to vary $T_{\infty}$ so that $\sigma_{\theta}^{*}(1)$ would never exceed a maximum 

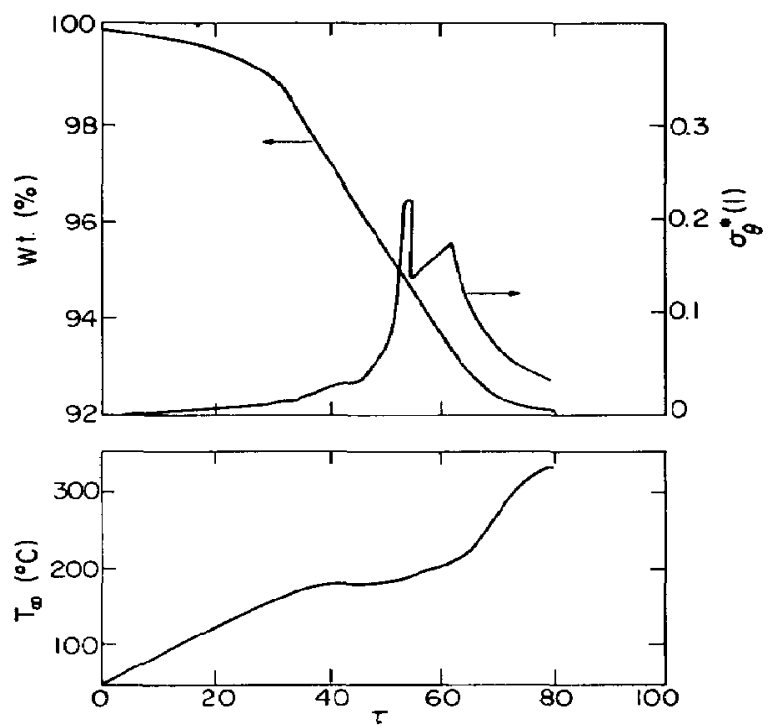

Fig. 8. Binder removal by rate-controlled extraction method: weight loss, tangential stress, and oven temperature as functions of time.

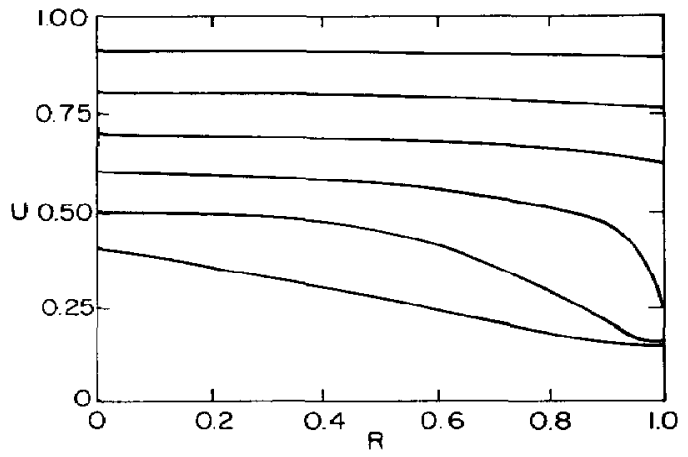

Fig. 9 Liquid saturation profiles for binder removal by ratecontrolled extraction method.

critical value at which rupture or failure would be expected to occur. The liquid saturation profiles for this case are shown in Fig. 9, displaying the undesirable gradients for $0.45 \leqslant U(0) \leqslant 0.6$ that result in the generation of large tensile stresses that cause microstructural damage. Thus, a somewhat arbitrary temperature-time profile was specified for a binder removal process that does not explicitly include a consideration of the magnitude of stresses that occur and the time at which they are likely to occur. This can result in a process design that either (1) will cause damage to the piece or (2) will necessarily be very slow and thus extremely long in duration so that flatter liquid saturation profiles and therefore lower tensile stresses can be insured. A more direct comparison of this method with the two "isothermal" processes $\left(T_{\mathrm{f}}=230^{\circ} \mathrm{C}\right.$ and $\left.155^{\circ} \mathrm{C}\right)$ are given by curve (c) in Figs 10 and 7 for weight loss and surface tangential stress histories, respectively.

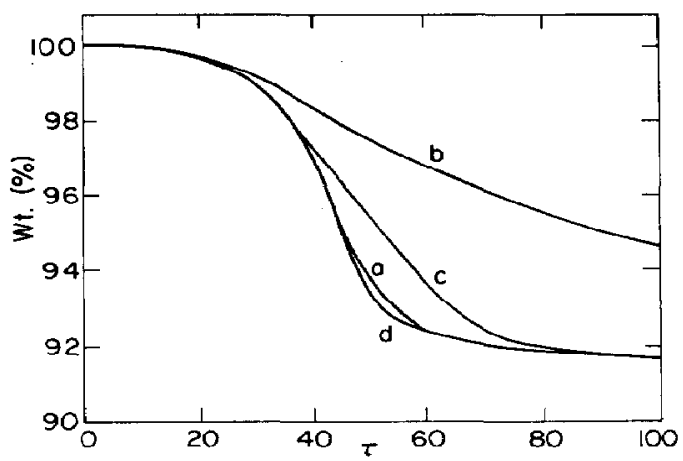

Fig. 10. Comparison of sample weight loss curves generated by various liquid removal methods.

A second application of this analysis considers the technique by which the liquid-phase viscosity is reduced in order to improve the binder removal process. This may be accomplished by employing a liquid that is a mixture of species of different molecular weights. This may be a polymer of high molecular weight (and thus high viscosity) used in combination with a polymer of the same type of lower molecular weight-resulting in a mixture with intermediate viscosity. Plasticizers can also be used to accomplish the same effect. Now, by eq. (2), a reduction in liquid viscosity should increase $v_{\mathrm{I}}$. Then, for a given $K_{\mathrm{L}}^{\mathrm{E}}$, which depends only upon the microstructure and local liquid content, and a given $P_{\mathrm{C}}$, which depends primarily upon the pore size distribution, a smaller value of $\mu_{\mathrm{L}}$ should result in a higher rate of liquid transport to the sample surface, thereby effectively eliminating liquid saturation gradients that arise for more viscous liquids. In this way, liquid from the interior of the body can more easily replenish liquid near the surface of the sample that has been lost to the external medium. A flatter liquid saturation profile would thus be more easily achieved, and internal stresses would therefore be reduced. To illustrate the effect, the "isothermal" conditions with $T_{\mathrm{f}}=230^{\circ} \mathrm{C}$ were repeated with a viscosity of $\mu_{\mathrm{L}} / 10$. (How this might be accomplished physically is not entirely clear but was chosen for simulation here to illustrate the importance of reduced liquid viscosity.) The liquid saturation profiles are given in Fig. 11 and are much flatter than those in Fig. 4. Further, the corresponding weight loss and $\sigma_{\theta}^{*}(1)$ versus dimensionless time curves are shown as curve (d) in Fig. 10 and curve (c) in Fig. 7. The weight loss curves (a) and (c) in Fig. 10 show little difference from one another, but the surface tangential stress profiles (a) and (c) in Fig. 7 are markedly different, indicating the importance of liquid viscosity upon internal stress generation during the removal of kiquid from a porous granular body.

The results presented in this paper are of relevance to commercially significant processes that involve partially saturated granular materials, such as binder removal from ceramic compacts. The theoretical model described here was seen to predict experi- 


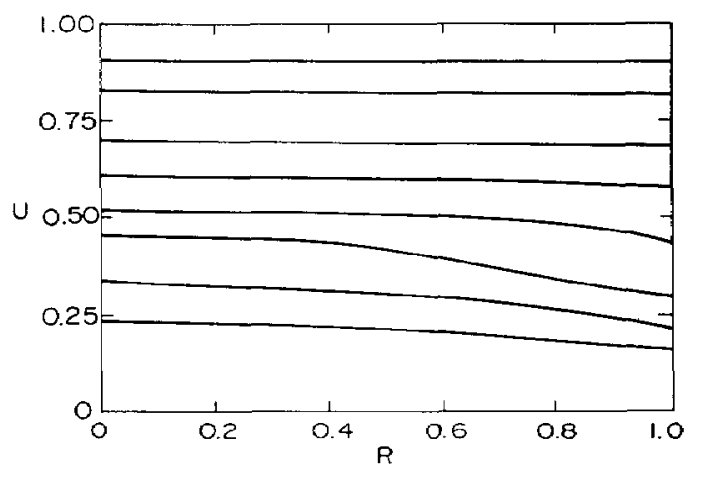

Fig. 11. Liquid saturation profiles for isothermal heating conditions $\left(T_{\mathrm{f}}=230^{\circ} \mathrm{C}\right)$ for a ten-fold liquid viscosity decrease.

mental results with sufficient accuracy that it could then be applied in the analysis of existing binder removal schemes. The calculations were performed for a particular system, submicron $\alpha-\mathrm{Al}_{2} \mathrm{O}_{3}$ particles in paraffin wax, but the analysis has yielded results that may be applied (at least qualitatively) to any binder removal process. For example, the relative rates of capillary liquid flow toward the sample surface and of convective mass transfer from the surface to the external medium are shown to significantly influence the magnitude of internal tensile stresses generated during binder removal. Further, the microstructure of the body (i.e., the spatial arrangement of solid grains and the geometry and topology of the interstitial pore network) directly influences the transport processes taking place during binder removal. These considerations are important in the design of a successful ceramic fabrication process. This paper represents a step in the direction of providing a more fundamental understanding of the important factors involved and establishes some guidelines for the rational design of an improved binder removal process.

Acknowledgements - This work was supported by the Air Force Office of Scientific Research/Defence Advanced Research Projects Agency under Grant No. AFOSR-87-0114 and by the IBM Corporation as part of a block grant for the microdesigning of ceramics and ceramic/polymer composites.

\section{NOTATION}

$\alpha_{n}^{0}$
$A_{1}, \ldots$
$C_{\mathrm{f}}{ }^{A_{10}}$
$C^{\prime}(0)$
${ }_{n} C_{k}$
$C_{\mathrm{p}}$
$C_{\mathrm{pG}}$
$C_{\mathrm{pG} 1}$
$C_{\mathrm{pGN}}$
$C_{\mathrm{pL}}$
$C_{\mathrm{pS}}$

defined by eq. (23)

defined by eqs (50)-(59)

molar concentration of ambient gas, $\mathrm{kgmol} / \mathrm{m}^{3}$

defined by eq. (15)

binomial expansion coefficient

total sample heat capacity, $\mathbf{J} / \mathbf{k g}-\mathrm{K}$ total gas phase heat capacity, $\mathbf{J} / \mathbf{k g}-\mathbf{K}$ gaseous paraffin heat capacity, $\mathrm{J} / \mathbf{k g}-\mathrm{K}$ gaseous nitrogen heat capacity, J/kg-K liquid phase heat capacity, $\mathrm{J} / \mathrm{kg}-\mathrm{K}$ solid phase heat capacity, J/kg-K

\section{$d_{\mathrm{p}}$ \\ $D_{\text {ABr }}$ \\ $D_{e, j}$ \\ $D_{j, \mathrm{~m}}$ \\ $D_{\mathbf{K}_{j}}$ \\ $D^{\mathbf{E}}$ \\ E \\ $F(k)$ \\ $f(r)$ \\ $g$}

$G(k)$

$h_{\mathrm{e}}$

$h_{\mathrm{m}}$

$H(k)$

$I_{m n o}$

$k$

$k_{F}$

$k_{\mathrm{G}}$

$k_{G 1}$

$k_{\mathrm{GN}}$

$k_{\mathrm{L}}$

$k_{\mathrm{s}}$

$k_{\mathrm{T}}$

$k_{\varphi}$

$\bar{k}_{\mathrm{L}, i}$

$K_{\mathbf{G}}^{\mathbf{E}}$

$K_{\mathrm{E}}^{\mathrm{E}}$

$K_{\mathbf{T}}^{\mathbf{E}}$

$m$

$\dot{m}_{\mathrm{c}}$

$\dot{m}_{i}$

$M_{1}, M_{2}$,

$\dot{M}_{\mathrm{S}}$

$n$

$N$

$N_{k}, N_{j}$

$P_{\text {atm }}$

$P_{\mathrm{C}}$

$P_{G}$

$P_{\mathrm{L}}$

$P_{1}^{0}$

$r$

$r_{i}$

$r_{\kappa}$

$r_{\mathrm{p}}$
$R$
$R_{\mathrm{gas}}$ sample diameter, $\mathrm{m}$

diffusion coefficient in ambient gas, $\mathrm{m}^{2} / \mathrm{s}$ effective local diffusion coefficient, $\mathrm{m}^{2} / \mathrm{s}$ diffusion coefficient of component $j$ through mixture, $\mathrm{m}^{2} / \mathrm{s}$

Knudsen diffusion coefficient of $j$ th component, $\mathrm{m}^{2} / \mathrm{s}$

effective diffusivity, $\mathrm{m}^{2} / \mathrm{s}$

Young's modulus of the bone-dry porous body

distribution function defined by eq. (18)

pore size distribution function

gravitational acceleration, $\mathrm{m} / \mathrm{s}^{2}$

function defined by eq. (20)

heat transfer coefficient, $W / m^{2}-K$

mass transfer coefficient, $\mathrm{kg} / \mathrm{m}^{2}$-s

function defined by eq. (21)

integral defined by eq. (24)

Boltzmann's constant, $\mathrm{J} / \mathrm{K}$

fluid phase thermal conductivity, $W / m-K$ total gas phase thermal conductivity, $\mathrm{W} / \mathrm{m}-\mathrm{K}$

gaseous paraffin thermal conductivity, $\mathrm{W} / \mathrm{m}-\mathrm{K}$

nitrogen thermal conductivity, W/m-K

liquid phase thermal conductivity,

W/m-K

solid phase thermal conductivity,

$\mathbf{W} / \mathbf{m}-\mathbf{K}$

defined as $\partial P_{\mathrm{C}} / \partial T, \mathrm{~Pa} / \mathrm{K}$

defined as $\partial P_{C} / \partial \varphi_{L}, \mathbf{P a}$

representative local transport coefficient, $\mathrm{m}^{2}$

effective gas permeability, $\mathrm{m}^{2}$

effective liquid permeability, $\mathrm{m}^{2}$

effective thermal conductivity, $\mathrm{W} / \mathrm{m}-\mathrm{K}$

index in eq. (24)

total local volumetric evaporation rate, $\mathrm{kg} / \mathrm{m}^{3}$-s

local volumetric evaporation rate of $i$ th component, $\mathrm{kg} / \mathrm{m}^{3}$-s

molecular weight of components 1,2 , and

$j$, respectively, $\mathrm{kg} / \mathrm{kgmol}$

total surface flux, $\mathrm{kg} / \mathrm{m}^{2}-\mathrm{s}$

index in eq. (24)

number of gas-phase components

flux of components $k, j$, respectively, $\mathrm{kg} / \mathrm{m}^{2}-\mathrm{s}$

ambient gas pressure, $\mathbf{P a}$

capillary pressure, $\mathbf{P a}$

local gas-phase pressure, $\mathrm{Pa}$

local liquid-phase pressure, $\mathrm{Pa}$

vapor pressure, $\mathbf{P a}$

radial position coordinate, $m$

radius of $i$ th pore, $m$

reaction rate term from decomposition of single-component liquid to form " $\kappa$ th" gas-phase species, $\mathrm{kg} / \mathrm{m}^{3}-\mathrm{s}$

sample radius, $\mathrm{m}$

dimensionless radial position coordinate gas constant, $\mathrm{m}^{3}-\mathrm{Pa} / \mathrm{kgmol}-\mathrm{K}$ 
$v$

$\rho$

$\rho_{\mathbf{G}}$

$\rho_{\mathrm{Gi}}$

$\rho_{\mathrm{GiO}}$

$\rho_{\mathrm{GN}}$

$\rho_{\mathrm{GNO}}$

$\rho_{\mathbf{L}}$

$\rho_{\mathbf{L}, \text { ref }}$

$\rho_{\mathrm{s}}$

$\sigma$

$\sigma$

$\sigma_{\theta}$

$\sigma$

$\bar{\sigma}$

$\sigma_{\mathbf{E}}$

$\tau$

$\varphi_{\mathbf{G}}$

$\varphi_{\mathbf{G} i}$

$\varphi_{\mathbf{L}}$

$\varphi_{\mathbf{L C}}$

$\varphi_{\text {LO }}$

$\varphi_{\mathbf{S}}$ summation index in eq. (6)

time, $s$

temperature, $\mathbf{K}$

critical temperature, $\mathbf{K}$

final oven temperature, $K$

reduced temperature

reference temperature, $K$

initial oven temperature, $K$

instantaneous oven temperature, $K$

radial displacement, $m$

dimensionless liquid content

local gas velocity, $\mathrm{m} / \mathrm{s}$

local liquid velocity, $\mathrm{m} / \mathrm{s}$

gas flow rate in furnace far from sample, $\mathrm{m} / \mathrm{s}$

dimensionless nitrogen gas density

mass fraction of $i$ th component at sample surface

mass fraction of $i$ th component far from sample

gas phase mole fraction of components $k$, $j, l$ and $N$, respectively

pore coordination number

particle coordination number

defined as $k_{\mathrm{s}} / k_{\mathrm{F}}$

thermal diffusivity; $\mathrm{m}^{2} / \mathrm{s}$

Dirac delta function

latent heat of vaporization, $J / \mathrm{kg}$

heat of reaction for $k \mathrm{th}$ reaction, $\mathrm{J} / \mathrm{kg}$

linear free strain

defined by eqs (14) and (15)

dimensionless temperature

summation index in eqs (4) and (5)

total gas viscosity, $\mathbf{P a - s}$

gaseous paraffin viscosity, Pa-s

gaseous nitrogen viscosity, $\mathrm{Pa}-\mathrm{s}$

liquid viscosity, $\mathrm{Pa}-\mathrm{s}$

Poisson ratio

total sample density, $\mathrm{kg} / \mathrm{m}^{3}$

total gas-phase density, $\mathrm{kg} / \mathrm{m}^{3}$

density of ith gas component, $\mathrm{kg} / \mathrm{m}^{3}$

initial density of $i$ th component, $\mathrm{kg} / \mathrm{m}^{3}$

nitrogen gas density, $\mathrm{kg} / \mathrm{m}^{3}$

initial nitrogen gas density, $\mathrm{kg} / \mathrm{m}^{3}$

liquid density, $\mathrm{kg} / \mathrm{m}^{3}$

reference liquid density, $\mathrm{kg} / \mathrm{m}^{3}$

solid-phase density, $\mathrm{kg} / \mathrm{m}^{3}$

surface tension, Pa-m

radial stress component, $\mathbf{P a}$

tangential stress component, $\mathbf{P a}$

net local stress tensor, Pa

volume-averaged $\sigma_{E}$, defined by eq.

(35), $\mathrm{Pa}$

effective local stress tensor, $\mathrm{Pa}$

dimensionless time

total local gas volume fraction

local volume fraction of ith component

local liquid volume fraction

critical liquid volume fraction

initial liquid volume fraction

solid volume fraction
$\begin{array}{ll}\Phi & \text { defined as } 1-\varphi_{\mathrm{S}} \\ & \text { second-order unit tensor }\end{array}$

\section{REFERENCES}

Batchelor, G. K. and O'Brien, R. W., 1977, Thermal or electrical conduction through a granular material. Proc. $R$. Soc. Lond. A 355, 313-333.

Berger, D. and Pei, D. C. T., 1973, Drying of hygroscopic capillary porous solids-a theoretical approach. Int. $J$. Heat Mass Transfer 16, 293-302.

Bird, R. B., Stewart, W. E. and Lightfoot, E. N., 1960, Transport Phenomena. John Wiley \& Sons, New York.

Carnahan, B., Luther, H. A. and Wilkes, J. O., 1969, Applied Numerical Methods. John Wiley \& Sons, New York.

Ceaglske, N. H. and Hougen, O. A., 1937, Drying granular solids. Ind. Engng Chem. 29, 805-813.

Chatzis, I. and Dullien, F. A. L., 1985, The modeling of mercury porosimetry and the relative permeability of mercury in sandstones using percolation theory. Int. Chem. Engng 25, 47-66.

DeWiest, R. J. M. (Ed.), 1969, Flow Through Porous Media. Academic Press, New York.

Dullien, F. A. L., 1979, Porous Media: Fluid Transport and Pore Structure. Academic Press, New York.

Gurr, C. G., Marshall, T. J. and Hutton, J. T., 1952, Movement of water in soil due to a temperature gradient. Soil Sci. 74, 335-348.

Johnsson, A., Carlstrom, D., Hermansson, L. and Carlsson, R., 1984, Rate-controlled thermal extraction of organic binders from injection-molded bodies. $\mathrm{Adv}$. $\mathrm{Ce}$ ram. 9, 241-245.

Luikov, A. V., 1966, Heat and Mass Transfer in CapillaryPorous Bodies. Pergamon Press, New York.

McTigue, D. F., Wilson, R. K. and Nunziato, J. W., 1983, An effective stress principle for partially saturated granular media. In Mechanics of Granular Materials: New Model and Constitutive Relations (Edited by Jenkins, J. T. and Satake, M.), pp. 195-210. Elsevier, Amsterdam.

Mohanty, K. K., Ottino, J. M. and Davis, H. T., 1982, Reaction and transport in disordered composite media: introduction of percolation concepts. Chem. Engng Sci. 37, 905-924.

Nozad, I., Carbonell, R. G. and Whitaker, S., 1985a, Heat conduction in multiphase systems. I. Theory and experiment for two-phase systems. Chem. Engng Sci. 40, 843-855.

Nozad, I., Carbonell, R. G. and Whitaker, S., 1985b, Heat conduction in multiphase systems. II. Experimental method and results for three-phase systems. Chem. Engng Sci. 40, 857-863.

Plumb, O. A., Spolek, G. A. and Olmstead, B. A., 1985, Heat and mass transfer in wood during drying. Int. J. Heat Mass Transfer 28, 1669-1678.

Ramakrishnan, T. S. and Wasan, D. T., 1986, Effect of capillary number on the relative permcability function for two-phase flow in porous media. Powder Technol. 48, 99-124.

Reyes, S. and Jensen, K. F., 1985, Estimation of effective transport coefficients in porous solids based on percolation concepts. Chem. Engng Sci. 40, 1723-1734.

Reyes, S. and Jensen, K. F., 1986a, Percolation concepts in modelling of gas-solid reactions. I. Application to char gasification in the kinetic regime. Chem. Engng Sci. 41, 333-343.

Reyes, S. and Jensen, K. F., 1986b, Percolation concepts in modelling of gas-solid reactions. II. Application to char gasification in the diffusion regime. Chem. Engng Sci. 41, 345-354.

Rhee, D.-J., Stangle, G. C. and Aksay, I. A., 1990, Binder rcmoval from ceramic/polymer composites. J. Am. Ceram. Sac. (manuscript in preparation).

Ridgeway, K. and Tarbuk, K. J., 1967, The random packing of spheres. Br. chem. Engng 12, 384-388. 
Scheidegger, A. E., 1954, J. appl. Phys. 25, 994 (1954); as quoted in Van Brakel (1975).

Scheidegger, A. E., 1974, The Physics of Flow Through Porous Media, 3rd Edn. University of Toronto Press, Toronto.

Sherrington, P. J. and Oliver, R., 1981, Granulation. Heyden $\&$ Son, London.

Sherwood, T. K., 1931, Application of the theoretical diffusion equations to the drying of solids. Trans. Am. Inst. Chem. Engrs 27, 190-202.

Stangle, G. C., Prime, R. B., Rhee, D.-J., Seferis, J. C. and Aksay, I. A., 1989, The relative importance of thermal cracking and reforming during binder removal from ceramic/polymer composites. In SPE ANTEC 89 Conference Proceedings, pp. 1066-1069.
Stinchcombe, R. B., 1974, Conductivity and spin-wave stiffness in disordered systems an exactly soluble model. $J$. Phys. C: Solid State Phys. 7, 179-203.

Timoshenko, S. P. and Goodier, J. N., 1970, Theory of Elasticity. McGraw-Hill, New York.

Van Brakel, J., 1975, Pore space models for transport phenomena in porous media: review and evaluation with special emphasis on capillary liquid transport. Powder Technol. 11, 205-236.

Whitaker, S., 1977, Simultaneous heat, mass and momentum transfer in porous media: a theory of drying. Adv. Heat Transfer 13, 119-203.

Whitaker, S. and Chou, W. T. H., 1983, Drying granular porous media - theory and experiment. Drying Technol. 1, 3-33. 